\title{
Nondestructive Integrated CT-XRD Method for Research on Hydrated Cement System
}

\author{
T. Sugiyama \\ Environmental Materials Engineering Laboratory, Faculty of Engineering, Hokkaido University \\ T. Hitomi \\ Construction System and Materials Department, Obayashi Corporation \\ K. Kajiwara \\ Industrial Application Division, Japan Synchrotron Radiation Research Institute
}

\begin{abstract}
A nondestructive integrated CT-XRD method has been developed and used to study hydrated cement system. In this research, a beam line $(\mathrm{BL})$ at the third-generation synchrotron radiation facility, SPring-8, in Japan, was used. First, X-ray computed tomography (CT) was employed to obtain three-dimensional (3D) images and select a region of interest $(\mathrm{ROI})$ in a given plane section of the hardened cement paste. Then, $\mathrm{X}$-ray diffraction analysis (XRD) was conducted on the specified region. These operations were implemented in situ without the removal of specimen from the stage inside the BL. The hardened cement paste was precracked and then leached by continuous water flow through the specimen, and the integrated CT-XRD method was conducted before and after the leaching test. In this way, the change in the hydrated cement system was characterized over time using the same specimen. CT observation provides the location of cracks and air voids as well as high and low density substances present in the hydrated cement system. ROI is arbitrarily determined at a set of coordinates at which one likes to evaluate the change in the cement system using XRD. This newly developed technique enables the evaluation of the presence of calcium hydroxide (portlandite) over time and space.
\end{abstract}

\section{INTRODUCTION}

Concrete has been a promising candidate in use for the construction of radioactive waste disposal facilities in Japan. The service life is expected to exceed over 10,000 years, and the long-term stability of a radioactive waste repository is indispensable during the intended operation period. At present, for a subsurface low-level waste disposal facility in Japan, geological repository with a given engineered barrier system is planned for construction of 50-100 m underground with horizontal tunnels about $10 \mathrm{~m}$ in diameter (Niwase, Hironaga, \& Tsuji, 2006). The barrier system consists of a multilayer system with compacted bentonite and cement-based materials (Sugiyama \& Tsuji, 2008). The compacted bentonite is used as a buffer material for its low water permeability, self-sealing characteristics due to its swelling capacity, and the adsorption of radionuclides. On the contrary, several types of cement based materials such as low-alkali binders and highly durable cementitious materials are being considered for application to the multilayer system. Under these conditions, deterioration of the hardened cement matrix due to the ground water is a primarily concern. This is because of the increased risk of the failure of the concrete. It is said that a hydrated cement system undergoes a dissolution of the hydration product due to continuous contact with water flow, followed by a reduction in structural performance (Carde \& Francois, 1997; Delegrave, Gerand, \& Marchand, 1997; Haga, Shibata, Hironaga, Tanaka, \& Nagasaki, 2005). In fact, the stability of the multilayer system employed in radioactive waste disposal facilities is predicted through analysis, but the accuracy of the simulation is hardly acceptable. As a result, a large volume of cementitious materials is required to satisfy the necessary safety level. This, in turn, results in increased construction cost. Accordingly, from an economic viewpoint as well as phenomenological aspect, it is necessary to increase the accuracy of the simulation. To achieve this goal, one factor is to estimate the depth of dissolution front as precisely as possible. In addition, the degraded region in the hydrated cement system due to groundwater attack needs to be accurately predicted. Thus, it is necessary to develop a more accurate experimental technique, so that the multiplicative effect is achieved through an enhanced model.

With conventional experiments, the detectable order of measurement for the dissolution front ranges from 1 to $10 \mathrm{~mm}$ in precision at most, and it is given merely 
in one dimension. In addition, although the use of electron probe X-ray micro-analyzer (EPMA) permits measurement on the order of $0.1 \mathrm{~mm}$, chemical compounds cannot be identified - only the profile of atoms. Considering the slow kinetic process of hydrate leaching due to water attack, the period of experiment becomes lengthy, and this period is increased further when testing concrete with lower water to cement ratios. Another drawback with conventional techniques is the fact, that to measure the degrading region, the specimen must be destroyed each time during the experiment. This limits the ability to understand the change in hydrated cement system over longer periods.

\section{BACKGROUND}

Our research group has developed a nondestructive technique to study the microstructure of hardened cement paste using X-ray $\mathrm{CT}$ at a synchrotron radiation facility, SPring-8, in Japan (Hitomi \& Kataoka, 2011; Hitomi, Mita, Saito, \& Takeda, 2004; Promentilla, Sugiyama, Hitomi, \& Takeda, 2008, 2009; Sugiyama, Promentilla, Hitomi, \& Takeda, 2010). A brief introduction of the primary research works is provided hereafter.

Promentilla et al. attempted to directly evaluate the pore structure-transport parameter from microtomographic images at a spatial resolution of $0.5 \mu \mathrm{m}$. Tortuosity, as being influenced by the $3 \mathrm{D}$ pore microgeometry, is an important physical quantity that was calculated with random walk simulation (RWS). They concluded that, without resorting to any assumption of pore geometry, the tortuosity associated with the percolated pore space seems to reflect the pore morphology that mainly controls the transport properties in cement pastes.

Sugiyama et al. investigated deteriorated mortars and cement pastes $(\mathrm{w} / \mathrm{c}=0.5)$ with $\mathrm{CaO} / \mathrm{SiO}_{2}$ molar ratio less than 2.0 using the leaching test. Nondestructive $3 \mathrm{D}$ imaging of the internal microstructure of the deteriorated cement matrix was performed. The microtomographic images successfully visualized increased pore space in the deteriorated cement matrices, with an effective porosity ranging from 0.31 to 0.38 . In addition, the diffusion tortuosity in the pore space derived from the RWS was also evaluated as a pore structure-transport parameter. Indications suggest that the deterioration of a cement matrix due primarily to the dissolution of portlandite decreases the diffusion tortuosity to a single digit, meaning that the pore connectivity becomes larger at the submicron scale.

Hitomi et al. directly observed self-healing phenomena of a crack in OPC mortar. Compared with high-strength mortar (HSC), OPC exhibited self-healing in the crack as well as the formation of new cracks. On the contrary, the self-healing was limited, but the alteration of cement paste in the vicinity of the crack was observed for HSC. In addition, different crack geometry in highstrength mortar was found.

It is understood that pores or air voids and high/ low density substances are clearly segmented by the X-ray CT method (Bentz et al., 2000; Gallucci, Scrivener, Groso, Stampanoni, \& Margaritondo, 2007; Landis \& Nagy, 2000; Promentilla \& Sugiyama, 2010). However, it may be said that, even using the X-ray CT in the synchrotron radiation facility SPring-8 in Japan, the distribution of a mineral in three dimensions is difficult to evaluate for a hydrated cement system. This limitation led to the motivation to develop a new method. The analysis of the linear attenuation coefficient in the histogram leaves room for further study to identify minerals. However, differences in the X-ray absorption characteristic of minerals present in a hydrated cement system are small, resulting in lowered contrast in a CT image. A crystalline mineral is formed with different crystal structures resulting in different diffraction spectra. In this method, the authors saw the advantage of $\mathrm{X}$-ray diffraction technique and proposed to couple CT and XRD measurements. Then, we started the development of a nondestructive integrated CT-XRD method.

\section{NONDESTRUCTIVE INTEGRATED CT-XRD METHOD}

The nondestructive INTEGRATED CT-XRD method has been developed at BL28B2, in the synchrotron radiation facility, SPring-8, in Japan (Hitomi, Kajiwara, \& Sugiyama 2013; Ikeda, Sugiyama, Hitomi, \& Kajiwara, 2013; Kajiwara, Hitomi, \& Sugiyama, 2013). The specified beam line (BL)employs whiteX-ray diffraction, which is one of the unique features of this method. A white X-ray covers a wide range of wave length zone. Therefore, with white X-ray as the incident X-ray a diffraction spectrum is obtained without scanning the angles of a detector and sample. In this way, so-called energy dispersive $\mathrm{X}$-ray diffraction measurement can be conducted using white X-ray.

The procedure for the measurement of the nondestructive integrated CT-XRD method is shown in Figure 1. A sample sits on the stage in the BL hutch. First, CT measurement is carried out followed by reconstruction to obtain microtomographic images. Then, the region of interest (ROI) in a given crosssection of the sample is determined. Finally, XRD is measured targeting the ROI.

Aschematic illustration of the nondestructive integrated CT-XRD method is shown in Figure 2. 
Sample sitting on the stage inside Beam Line hutch

CT measurement

- Reconstruction

- Determination of Region of Interest (ROI)

XRD measurement

-XRD targeted at the ROI

Removal of sample

Figure 1. Flowchart of nondestructive integrated CT-XRD method.

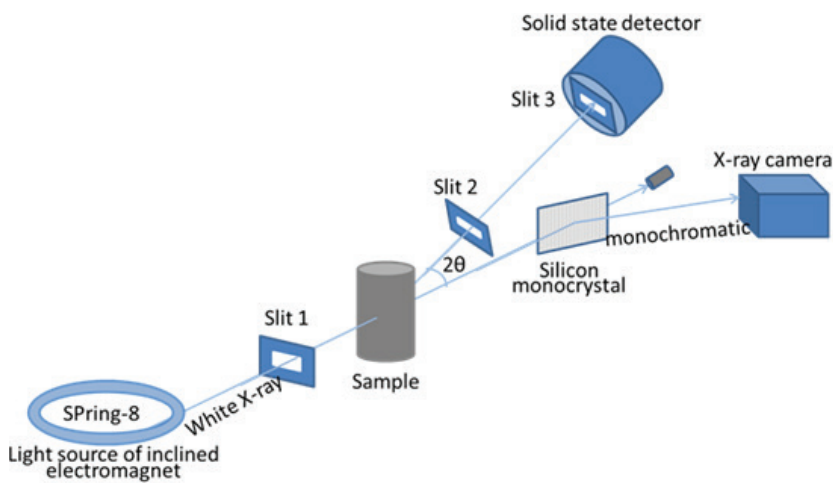

Figure 2. Schematic of the system for nondestructive Integrated CT-XRD method (Kajiwara et al., 2013; Kajiwara, Hitomi, Sugiyama, \& Sato, 2014).

\subsection{X-ray CT}

Synchrotron white X-ray is applied to a hardened cement paste, and X-ray that transits the sample is outputted to a silicon monocrystal located in the downstream position. Through the silicon monocrystal, the X-ray with a characteristic X-ray energy is diffracted (monochromatic) and captured by X-ray camera. The reason why the $\mathrm{X}$-ray monochromatic extracted from the white $X$-ray is used for this CT measurement is for the enhancement of image contrast. In addition, it is intended that the extraction of the monochromatic is made in the downstream position, so that there is no need to change the relative position of the incident X-ray to the sample. In this way, the coordinate system in between CT measurement and XRD measurement is unified. The sample sits on the revolving stage and X-ray is applied during rotation of the sample. Transmission images of X-ray with different angles are obtained, followed by image reconstruction with back projection procedure. By observing the reconstructed image in three dimensions, the region of interest (ROI) can be determined for the XRD measurement.

\subsection{X-ray diffraction}

$\mathrm{X}$-ray diffraction spectrum is measured in the ROI. To extract the signal of the X-ray diffraction only in the ROI, various slits are used (see Figure 2). A slit in the upstream position (S1) and two slits in the downstream position (S2 and S3) are designated so as to obtain only signals of X-ray diffraction in a given space, called the gauge volume. The gauge volume must fit to the ROI. This is easily accomplished by the arrangement of the automatic stage because the coordinate system from the CT measurement corresponds to that in the XRD. Detector is used with a solid-state detector. Slits S2 and S3 and the detector are all in position on the arm of the detector tube. The angle of the arm (2 $\theta)$

that corresponds to the twice of the diffraction angle $(\theta)$ is fixed at $20^{\circ}$ during the measurement. Through the detector, the relationship of X-ray energy and the intensity of the diffracted $X$-ray is obtained. One may accustom to the relationship between the diffraction angle and the intensity of the X-ray obtained from a laboratory XRD machine with specified wavelength. This is converted using the relationship between X-ray energy and wavelength integrated with the Bragg equation, given below:

$$
2 \mathrm{~d} \sin \theta=n \lambda,
$$

where $d$ is the spacing between the planes, $\lambda$ is the wavelength of the incident wave, $n$ is a natural number and equal to 1 , and $\theta$ is the angle between the incident ray and the scattering planes.

\section{EXPERIMENT}

\subsection{Sample preparation}

Hardened cement paste was studied. For the binder, only ordinary Portland cement (OPC) was used. The water to cement ratio was 0.5 . A steel mold with $40 \mathrm{~mm} \times 40 \mathrm{~mm} \times 160 \mathrm{~mm}$ in size was used. After removal of the mold within $24 \mathrm{~h}$, the cement paste was cured in water for a period of 52 days. Then it was cut so as to obtain a cylinder-shaped specimen about $5 \mathrm{~mm}$ in diameter and $5 \mathrm{~mm}$ in the length. Aluminum tape was used to cover the cylinder and to attach plastic tubes at both ends. Next, a crack was induced by splitting the specimen. Later, water was supplied to the specimen through the tubes for carrying out the leaching test.

\subsection{Measurements}

The setup of the instruments and specimen is shown in Figure 3. The specimen was fixed on an aluminum pedestal on the precision revolving stage. The extracted energy of X-ray for CT measurement was $25 \mathrm{keV}$. About 900 projection views were obtained with an exposure time of $0.15 \mathrm{~s}$. One pixel size was 
$0.0074 \mathrm{~mm}$, and the number of pixels was 735 pixels. The overall field of view was about $5 \mathrm{~mm}$.

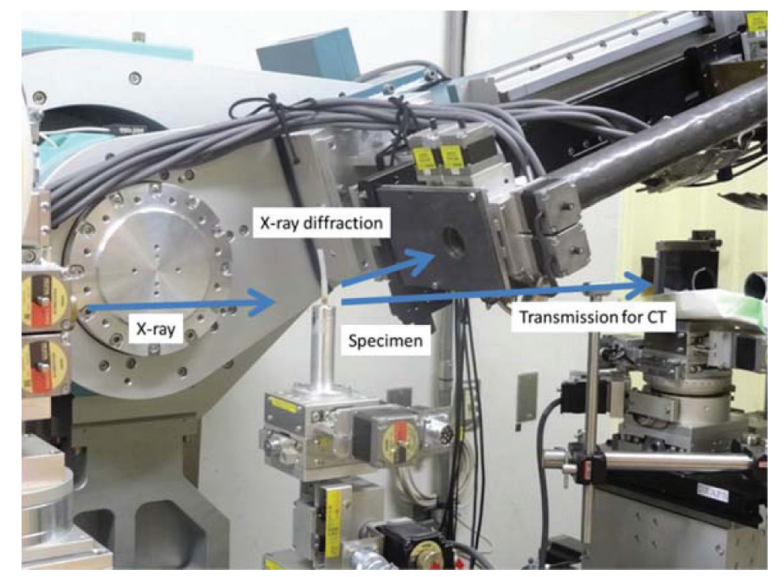

Figure 3. Picture of the system around the specimen in BL28B2, SPring-8.

The beam size was $0.05 \mathrm{~mm}$ in width and $0.15 \mathrm{~mm}$ in height. The angle of diffraction $(\theta)$ was fixed at $10^{\circ}$. The preset time was $300 \mathrm{~s}$. During measurement, the stage was rotated, so that the diffraction signal could be adequately obtained by the increased crystal grain. By doing so, the average diffraction chart was determined, characterizing the crystal grain present in the observation region.

\subsection{Leaching test}

As shown in Figure 4, the leaching test was carried out for cracked cement paste. A tubing pump with a microtube $2 \mathrm{~mm}$ in inner diameter was used. Pure water was used, and the flow rate was a constant $50 \mathrm{cc} / \mathrm{h}$. For the first week, the discharged water was collected to measure the calcium concentration. It was confirmed that the concentration of calcium ions increased in the first few days and then leveled off later. From the second week, water was circulated using a tank of $30 \mathrm{~L}$ in volume via the tube pump.

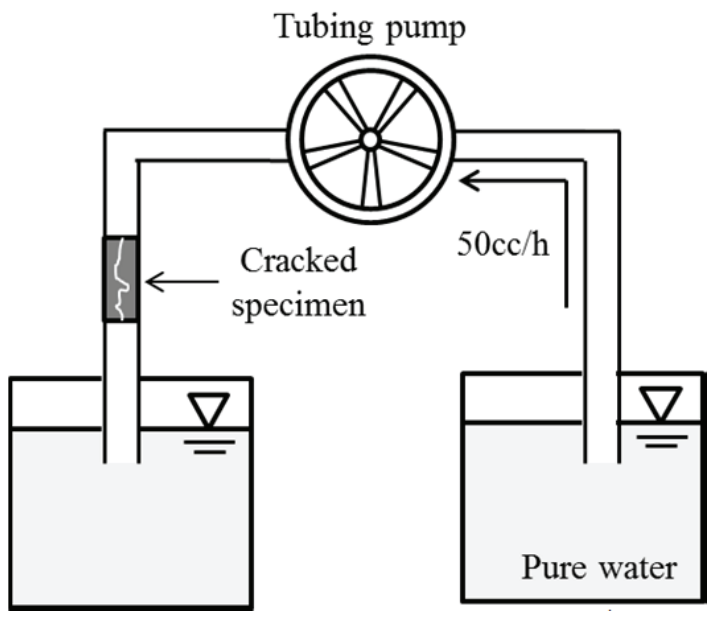

Figure 4. Water flow in cracked specimen during first week.
The procedure of the measurement by nondestructive integrated CT-XRD method and the dissolution of the hydrated cement system during the leaching test is shown in Figure 5.

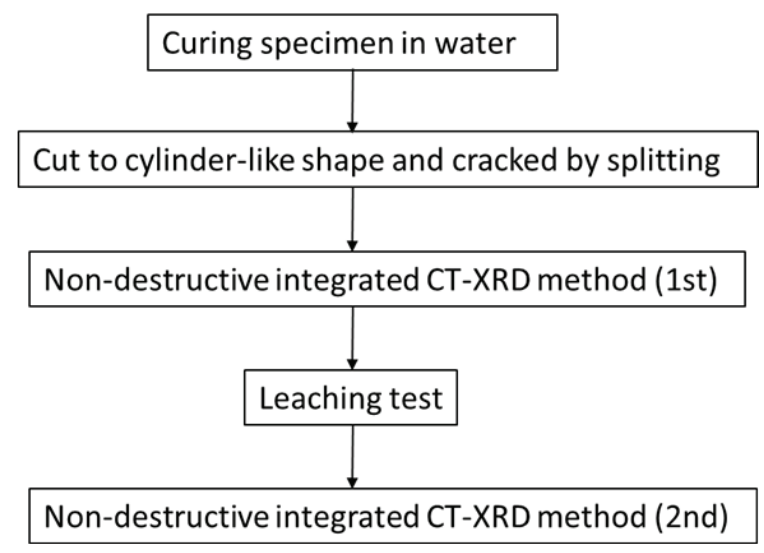

Figure 5. Flowchart of measurements.

\section{RESULTS AND DISCUSSION}

\subsection{Microtomographic image}

Figure 6 shows a microtomographic image of the cracked specimen before the leaching test. The crosssection is located $1.67 \mathrm{~mm}$ from the top surface of the specimen. This image is represented using gray scale value (GSV), where lower density substances exhibit lower GSV. Accordingly, the cracks appear as black, whereas solid regions appear as gray. The small white spots are unhydrated cement grains, which remain due to the cement paste's relatively higher density.

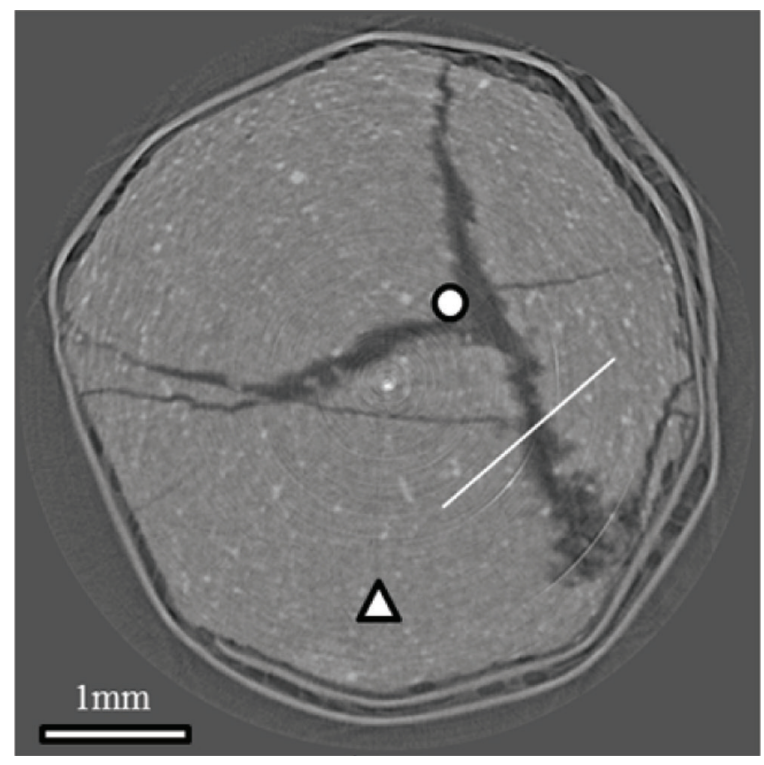

Figure 6. X-ray CT image (first). The markers indicate the position of the XRD measurements (circle: 50L1, triangle: 50L2). The solid white line is the location of the line profile of CT values. 
Multiple cracks were formed in the specimen despite the splitting action. This resulted from the angular shape of the cross-section and an eccentric stress induced in the specimen. Nevertheless, the microtomographic image shows clearly the cracks. By observing the image, two regions of interests were selected for XRD measurement. These are indicated with a circle and a triangle in Figure 6 . One is in the vicinity of the crack intersection where two primary cracks intersect (50L1: $\bigcirc)$. The other one is in the matrix a bit far from the crack edge (50L2: $\Delta$ ).

Figure 7 compares the line profiles of CT values before and after the leaching tests. The location from where the profile was taken is shown in Figure 6 (white straight line). Two distinct regions appeared in both line profiles, for example, high and low GSV. It is considered that lower GSV areas represent the crack. Then, assuming that the crack edge corresponds to the sudden drop in the GSV profile in Figure 7, one can estimate the crack width. In this way, the crack width is estimated as 0.22 and $0.62 \mathrm{~mm}$ for before and after the leaching test, respectively. This means that the crack opening became wider due to leaching. In addition, it is considered that higher GSV represents the solid phase. Before the leaching test, the GSV is higher than 150; however, the corresponding solid region has a slightly lower GSV afterwards. It can be said that even in solid regions, the density was reduced due to dissolution of the hydrated cement system.
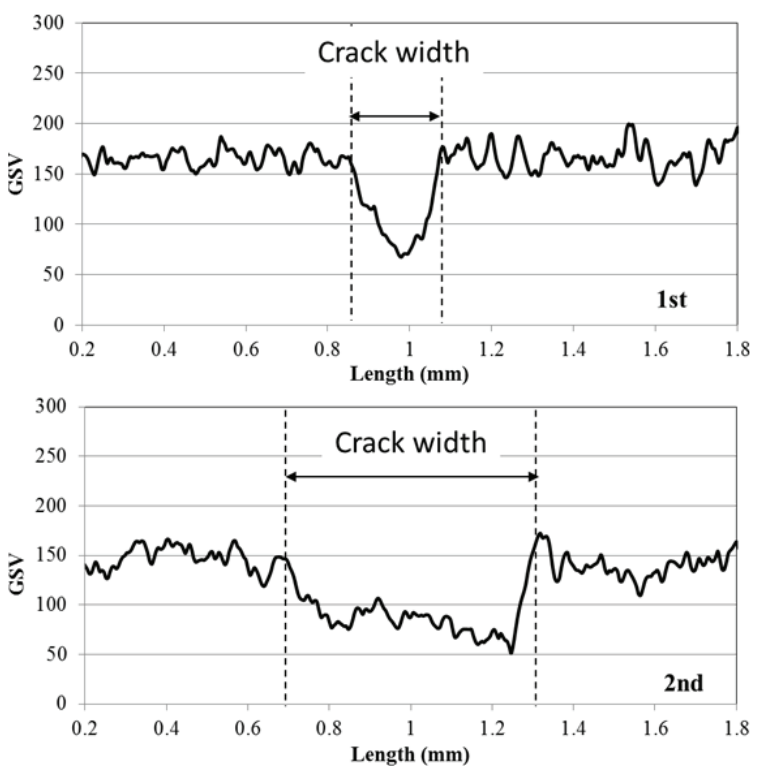

Figure 7. Results of line profiles of CT values.

\subsection{Diffraction spectrum}

Diffraction spectrums before and after the leaching testare shown in Figure 8. They were measured at two locations (50L1 and 50L2) as shown in the microtomographic image in Figure 6. It is noted that the diffraction spectrums after leaching test are also measured at corresponding coordinates in the microtomographic image. One of the primary hydration products from the reaction of $\mathrm{C}_{3} \mathrm{~S}$ and $\mathrm{C}_{2} \mathrm{~S}$ with $\mathrm{H}_{2} \mathrm{O}$ is calcium hydroxide. Thus, in Figure 8, the diffraction angles of portlandite are also provided as portlandite-ICSD. These data are taken from the Inorganic Crystal Structure Database (ICSD).
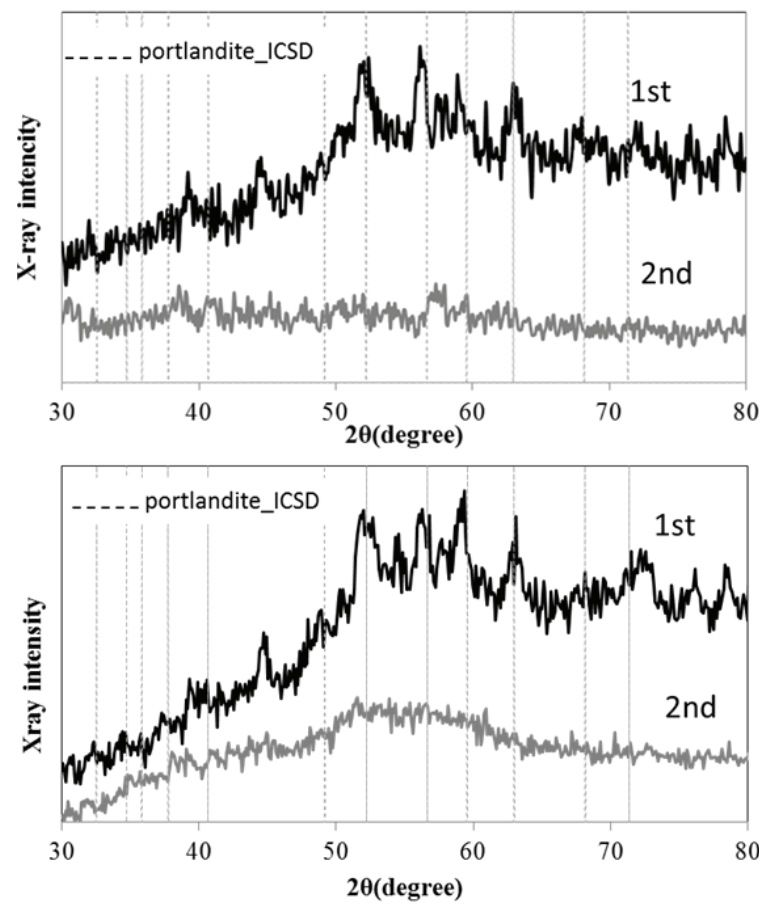

Figure 8. Diffraction spectrums: top figure shows the results in $50 \mathrm{~L} 1$. Bottom figure shows the results in 50L2.

In both locations $50 \mathrm{~L} 1$ and 50L2, the diffraction spectrums before leaching test exhibit distinct peaks at the given angles, which correspond to portlandite-ICSD. This means that the calcium hydroxide is present at both locations. On the contrary, the distinct peaks in the diffraction spectrums are hardly manifested after the leaching test. Therefore, the dissolution of calcium hydroxide occurred due to continuous water flow through the specimen. Cracks are normally considered to be a main passage for the penetration of water, resulting in more severe dissolution in nearby regions. In this research, calcium hydroxide was dissolved even in cement matrix (50L2) far from the crack. Therefore, the flow rate of $50 \mathrm{cc} / \mathrm{s}$ was severe enough to cause leaching across the entire specimen section.

The leaching behavior can make the hydrated matrix porous. This effect may be further studied using the diffraction spectrums in near future.

\section{CONCLUSIONS}

This article covers the introduction of the nondestructive integrated CT-XRD method, which was proposed as a new evaluation technique. To clarify its capability for 
the measurement of the physical and chemical nature of a hydrated cement system, a hardened cement paste of a water to cement ratio of 0.5 was tested. This method enabled the evaluation of calcium hydroxide presence over time and space.

\section{ACKNOWLEDGMENT}

Part of this research was funded by the Japan Society for the Promotion of Science (23360187). The synchrotron radiation experiments were performed at the BL28B2 in SPring-8 with the approval of the Japan Synchrotron Radiation Research Institute (JASRI) (2012B1282, 2013B1511, 2013B1594).

\section{REFERENCES}

Bentz, D. P., Quenard, D. A., Kunzel, H. M., Baruchel, J., Peyrin, F., Martys, N. S., \& Garboczi, E. J. (2000). Microstructure and transport properties of porous building materials. II: Three-dimensional $\mathrm{X}$-ray tomographic studies. Materials and Structures, 33, 147-153.

Carde, C., \& Francois, R. (1997). Effect of the leaching of calcium hydroxide fromcement paste on mechanical and physical properties. Cement and Concrete Research, 27(4), 539-550.

Delegrave, A., Gerand, B., \& Marchand, J. (1997). Modelling the calcium leaching mechanism in hydrated cement pastes, in: K. L. Scrivener \& J. F. Young (Eds.), Mechanisms of Chemical Degradation of Cement-Based Systems (pp. 38-49). London: E\&FN, SPON.

Gallucci, E., Scrivener, K., Groso, A., Stampanoni, M., \& Margaritondo, G. (2007). 3D experimental investigation of the microstructure of cement pastes using synchrotron X-ray microtomography. Cement and Concrete Research, 37, 360-368.

Haga, K., Shibata, M., Hironaga, M., Tanaka, S., \& Nagasaki, S. (2005). Change in pore structure and composition of hardened cement paste during the process of dissolution. Cement and Concrete Research, 35(5), 943-950.

Hitomi, T., Mita, Y., Saito, H., \& Takeda, N. (2004). Observation of fine structure of mortar using X-ray CT images at SPring-8. Proceedings of Annual Conference of Japan Concrete Institute, 26(1), 645-650 [in Japanese].

Hitomi, T., \& Kataoka, H. (2011). Study of the self-healing process of crack introduced mortar using $X$-ray CT. Proceedings of Annual Conference of Japan Concrete Institutes, 33(1), 1427-1432 [in Japanese].

Hitomi, T., Kajiwara, K., \& Sugiyama, T. (2013). Micro-observation of chemical compounds in mortar by non-destructive integrated CT-XRD method. 67th Annual Conference, Japan Cement Association, 80-81. [In Japanese].

Ikeda, S. Sugiyama, T., Hitomi, T., \& Kajiwara, K. (2013). Non-destructive integrated CT-XRD method for the observation of alteration of hardened cement paste with cracks due to water flow. 67th Annual Conference, Japan Cement Association. 84-85. [In Japanese].

Kajiwara, K., Hitomi, T., \& Sugiyama, T. (2013). Development of non-destructive integrated CT-XRD method for the evaluation of mineral distribution in cementitious materials. 67th Annual Conference, Japan Cement Association, 82-83. [In Japanese].

Kajiwara, K., Hitomi, T., Sugiyama, T., \& Sato M. (2014). Development of non-destructive integrated CT-XRD method for cementitious materials in BL28B2 of SPring-8, IUMRS-ICA, The Materials Research Society of Japan. (Accepted abstract).

Landis, E. N., \& Nagy, E. N. (2000). Threedimensional work of fracture for mortar in compression. Engineering Facture Mechanics, 65, 223-234.

Niwase, K., Hironaga, M., \& Tsuji, Y. (2006). Design of the concrete used for sub-surface LLW disposal facility. Concrete Journal, Japan Concrete Institutes, 44(2), 3-8 (in Japanese).

Promentilla, M. A. B., \& Sugiyama, T. (2010). X-Ray microtomography of mortars exposed to freezingthawing action. Journal of Advanced Concrete Technology, 8(2), 97-111.

Promentilla, M. A. B., Sugiyama, T., Hitomi, T., \& Takeda, N. (2008). Characterizing the 3D pore structure of hardened cement paste with synchrotron microtomography. Journal of Advanced Concrete Technology, 6(2), 273-286.

Promentilla, M. A. B., Sugiyama, T., Hitomi, T., \& Takeda, N. (2009). Quantification of tortuosity in hardened cement paste using synchrotron-based X-ray computed microtomography. Cement and Concrete Research, 39(6), 548-557.

Sugiyama, T., Promentilla, M. A. B., Hitomi, T., \& Takeda, N. (2010). Application of synchrotron microtomography for pore structure characterization of deteriorated cementitious materials due to leaching. Cement and Concrete Research, 40(8), 1265-1270.

Sugiyama, T., \& Tsuji, Y. (2008). Use of a migration technique to study alteration of compacted sand-bentonite mixture in contact with concrete. Physics and Chemistry of the Earth, 33, S276-S284. 